\title{
Quantitative Research on Product Form Based on Kansei Engineering
}

\author{
$\operatorname{Xin~} \mathbf{L i}^{1, *}$ \\ ${ }^{1}$ College of Electrical and Mechanical Engineering, Agricultural University of Hebei, Baoding, 071001, China
}

Received 29 September 2017; Accepted 8 February 2018

\begin{abstract}
In mature market competition, the evaluations of users on product design are significantly dependent on the perception of product forms. In order to study the mapping relationship between product form and perceptual image, a quantification algorithm model was proposed. Initially, samples and perceptual image word pairs were collected and selected on basis of helmet products. A score was given to each sample image by subjects, and the initial data were obtained. Subsequently, the data were pre-processed using the Leida criterion for rejecting the abnormal perceptual image. The samples were grouped into 12 categories by clustering analysis. Finally, a multidimensional scaling and linear regression analysis were utilized to generate the joint space map of perceptual attribute vectors and sample scattered points. The attribute value of the sample was obtained by calculating the distances from the scattered points to the projection points of the perceptual vector and the origin. Thereafter, the correspondence between the sample and perceptual words was obtained. Results demonstrate that the perceptual characteristics of words are more evident for the absolute attribute value larger than 2, rather than the absolute value less than 0.1 . The larger absolute attribute value shows that the sample form has more perceptual characteristics of the vector. The study provides an effective method for quantifying the perceptual characteristics of product form.
\end{abstract}

Keywords: Kansei engineering, Product form, Design orientation, Joint space map

\section{Introduction}

With the progress of the time, people's demands for products tend to be perceptual, and a user's evaluation of product form relies on the perception of product design. The formation of perceived image comes from people's cognition of a product. With their own senses, users can obtain visual and aesthetic perceptions through the stylistic information of a product. In addition, perception image contains value judgments using functions and cultural factors. The perceptual image of a product has become an important medium for conveying the psychological needs of users. In mature market competition, similar products of different brands have an apparent homogenization trend. Drawing the attention of users by the innovations on the practical functions of products is insufficient [1,2]. The perceptual experience of products has gradually become the decisive factor for users to purchase or not. Product form is the most direct way of reflecting perceptual factors, and product design has become important in the competitiveness of enterprises. Therefore, designers have studied users' basic perceptual rules of product form to have a potential psychological communication until effective communication $[3,4]$. The emergence and development of perceptual design address this demand. Perceptual design considers customers' perceptual needs in the product design process. However, most traditional studies on perceptual design are qualitative instead of quantitative, focusing on the discovery and

\footnotetext{
*E-mail address:lixin20131113@163.com

ISSN: 1791-2377 @ 2018 Eastern Macedonia and Thrace Institute of Technology. All rights reserved.

doi:10.25103/jestr.111.10
}

qualitative deduction in product design. The research and deduction of perception design quantification are significant innovations. Seeking the quantitative mapping between design elements and perceptual words is the focus and bottleneck of perceptual design research [5].

Thus, this study used the theory of Kansei engineering and the multidimensional scaling algorithm to study subjects' "perception" cognition rules of products [6,7,8]. Furthermore, we discussed the perception quantification method of product form and affirmed the relation between "perception" and product design, with the establishment of the effective mapping relation between perceptual words and design elements. This study has a reference value for the development of future product designs.

\section{State of the art}

Several scholars at home and abroad have performed algorithmic research on the perceptual quantization of product form. Zhao Y. Y., et al. [9] applied analytic hierarchy process and fuzzy evaluation method to study material image in product design. Le X. et al. [10] performed a perceptual evaluation of the service robot design based on the fuzzy Kano model. The fuzzy algorithm has a unique advantage in modeling with a high degree of ambiguity and can, thus, deal with ambiguous perceptual needs. Moreover, few or incomplete data are used during model development [11]. However, the current fuzzy algorithm cannot be used to establish a nonlinear system model.

Increasing studies have corroborated that the mapping relationship between perceptual needs and design variables is 
mainly established by statistical methods. Among the statistical methods, regression analysis has received significant attention from many scholars. Wang K. et al. [12] constructed quantification model I to solve the relationship between the form images of Chinese spirit bottles and the design elements. Du Y. et al. [13] studied the perceptual cognizance of college students and their form preference to backpacks. Factor, clustering, and type-I quantification analyses were used to analyze and deconstruct backpacks, thereby obtaining the relationship between form factors and images. Guo B.J. et al. [14] studied the influence of cheongsam collar and cardigan on the overall style through average, factor, and clustering analyses. Kuang J. et al. [15] used multivariate linear regression to establish a relationship model between design variables and perceptual responses. J. Kim et al. [16] used a general linear model to establish the quantitative relationship between design factor and perceptual dimension. C. Barnes et al. [17] proposed a decision support system based on principal component analysis and multiple regressions to provide guidelines for optimum perceptual satisfaction. Du H. M. et al. [18] used an explicit modeling approach based on vector fields to establish a relationship model between perceptual satisfaction and design variables. H. You et al. [19] developed a perceptual satisfaction model using quantification theory I to identify the significant or preferred design variables in a new product. The common feature of these methods is that the significant design variables should be screened first. Subsequently, the general linear model is used to apperceive perceptual satisfaction in the perceptual space. However, they rely on linear assumptions, which cannot effectively deal with non-linear relationships.

Therefore, on the basis of the cognitive needs of subjects, we discovered the similarity of sample forms and the relevance of image words by using several methods, such as Kansei engineering and multidimensional scaling. In addition, we demonstrated the non-linear relationship between design and perceptual variables, and established a perceptual joint space map of the samples. It vividly shows the relation between the samples and perceptual images with quantitative data.

The rest of this study is organized as follows. Section 3 introduces the method and research process of Kansei engineering. Taking helmet as an example for experiment design, we used the method of Kansei engineering in the questionnaire survey to obtain the initial data. Section 4 presents the statistical analysis of the data, with the establishment of the perceptual image space map of a helmet. Helmet form samples were obtained in the semantic rule database. The last section shows the relevant conclusions.

\section{Methodology}

\subsection{Kansei engineering}

Kansei engineering is a design technique that integrates perceptual image with engineering to explore the relationship between the perceptions of users and the product design features. Through the perceptual analysis of users, the perceptual images of products are qualitatively and quantitatively expressed, which can be transformed into design elements. Under the premise of practicality, it can design products that satisfy the users' psychological experience [2]. The operation of the quantitative Kansei engineering system relies on three databases.
(1) The perceptual database includes the adjectives that can express the users' perceptual needs of the products. For the target product, the set $\left\{w_{k} \mid k=1,2, \mathrm{~L}, k\right\}$ of perceptual words is used to express the perceptual needs of the target consumer group.

(2) The morphological database includes all forms of perceptual adjectives. The set $\left\{e_{d} \mid d=1,2, \mathrm{~L}, d\right\}$ is used to express the collection of design features of product forms.

(3) The image database is a statistical list of the relationship between perceptual words and design elements. For one or a group of perceptual needs, one or a group of specific design elements should satisfy these needs; one or a group of design elements can trigger one or more customer perceptual responses.

This study aimed to find the effective mapping relationship between the perceptual word set $\left\{w_{k} \mid k=1,2, \mathrm{~L}, k\right\}$ and the set $\left\{e_{d} \mid d=1,2, \mathrm{~L}, d\right\}$ of product design features. The research tools and statistical analysis methods of quantitative Kansei engineering include semantic differential method, multidimensional scaling, and clustering analysis. They are scientific methods of statistics, calculation, and analysis that use experiments to obtain the original data of psychological quantity rating when something is evaluated. The specific research process is as follows:

(1) Collection of samples and perceptual image vocabulary

Product images were collected from the Internet and books, and similar or duplicate ones were removed. Thereafter, we filtered out the images with a relatively single color, a universal material, and representative form.

Perceptual image words were used to describe the form style of products. Perceptual word pairs were extensively collected and classified. Subsequently, unfeasible pairs with similar connotations were excluded. The pairs of the most representative product intention were extracted to establish an effective semantic space.

(2) Data acquisition

The semantic differential method was used as the main experimental tool. The initial data were obtained using a questionnaire survey to examine the people's preference image of product form. The semantic differential method also known as SD method, is used to study the psychological images of subjects [15]. It is composed of an object, a rating scale, and subjects. The selection rating points of the scale should be odd, commonly 5 or 7 points [16]. The number of subjects should be at least 100 to obtain reliable information.

(3) Data analysis and summary

A statistical method was used to quantify the initial data of the questionnaire and screen out representative product samples and perceptual semantics. Subsequently, SPSS was applied to draw the joint space map of the sample and the semantic spaces. Through analysis, calculation, and verification, the mapping relationship between product form and intention words was obtained, which provided scientific and effective data for establishing the semantic rule database of product design.

\subsection{Application case}

Taking helmet as an example, we used Kansei engineering to study the corresponding relationship between the word pairs of perceptual image and product form. 


\subsubsection{Selection of image words}

Through related websites, magazines and conversations with helmet designers, salespersons and users, 60 perceptualimage adjectives that described helmet forms were listed. According to the suitability of word pairs to the samples, the respondents performed a five-point scoring system. Subsequently, the statistics on the scoring results was provided using the KJ (Kawakita Jiro) method and the SPSS software. The commonality larger than 0.5 indicated an ideal word pair - the significant average indicated a profoundly ideal word pair. Finally, 12 pairs of representative semantic adjectives were screened out (See Table 1).

Table 1. Screened perceptual vocabulary

\begin{tabular}{c|c}
\hline No. & Perceptual vocabulary \\
\hline 1 & Modern-Traditional \\
2 & Popularization_-Individuation \\
3 & Lively_-Inflexible \\
4 & Practical_-Decorative \\
5 & Kind_-Indifferent \\
6 & Mature_-Infantile \\
7 & Light_-Heavy \\
8 & Angular-Sleek \\
9 & Brief-Complicated \\
10 & Dynamic_-Static \\
11 & Safe-Dangerous \\
12 & Geometric_-Biomimetic \\
\hline
\end{tabular}

\subsubsection{Product sample selection}

Over 100 pictures of sports helmets of various forms were collected from the Internet and magazines, including the most popular helmets and concept helmets in recent years. After classification without considering colors and materials, 60 helmets with the most representative forms were finally selected. The pictures of these helmets were listed on the questionnaire (See Table 2).

\subsubsection{Questionnaire establishment and data acquisition}

The selected helmet samples and emotional words were used to establish a seven-point rating scale questionnaire. The measuring scale has seven levels, which indicate the deviation of words. The scores are $-3,-2,-1,0,1,2$, and 3 .

In terms of "popularity-personalization" words, the negative value indicates that the helmet form has the characteristics of "popularization," and the small value represents high popularity. The positive value indicates that the helmet form has the characteristics of "personalization," and the large value represents high popularization. 0 indicates uncertainty. Questionnaires were distributed to 34 juniors majoring in industrial design, 32 graduate students in industrial design, 32 helmet designers, and 32 helmet users. A total of 130 subjects completed the questionnaire and scored 60 helmets separately. Each subject was required to fill in the contents, including the personal information (age, gender, education, professional, and occupation) and questions.

\section{Result analysis and discussion}

\subsection{Preliminary statistics of objects}

The survey was sent out, and 130 questionnaires were collected, including 117 valid questionnaires, with a $90 \%$ effective rate. After the statistics of the 7-point rating scale was generated, the final survey model can be defined as follows.
$\left[\begin{array}{ccccc}A_{11}^{K} & A_{12}^{K} & A_{13}^{K} & \ldots & A_{1 J}^{K} \\ A_{21}^{K} & A_{22}^{K} & A_{23}^{K} & \ldots & A_{2 J}^{K} \\ & \ldots & & A_{i j}^{K} & \\ A_{I 1}^{K} & A_{I 2}^{K} & A_{I 3}^{K} & \ldots & A_{I J}^{K}\end{array}\right]$

Where $A_{i j}^{K}$ is the $\mathrm{j}$-th perceptual evaluation preference from $\mathrm{k}$ evaluators to the $\mathrm{i}$-th evaluation sample, $i$ is 60 , and $j$ is 12 .

Table 2. Screened helmet picture

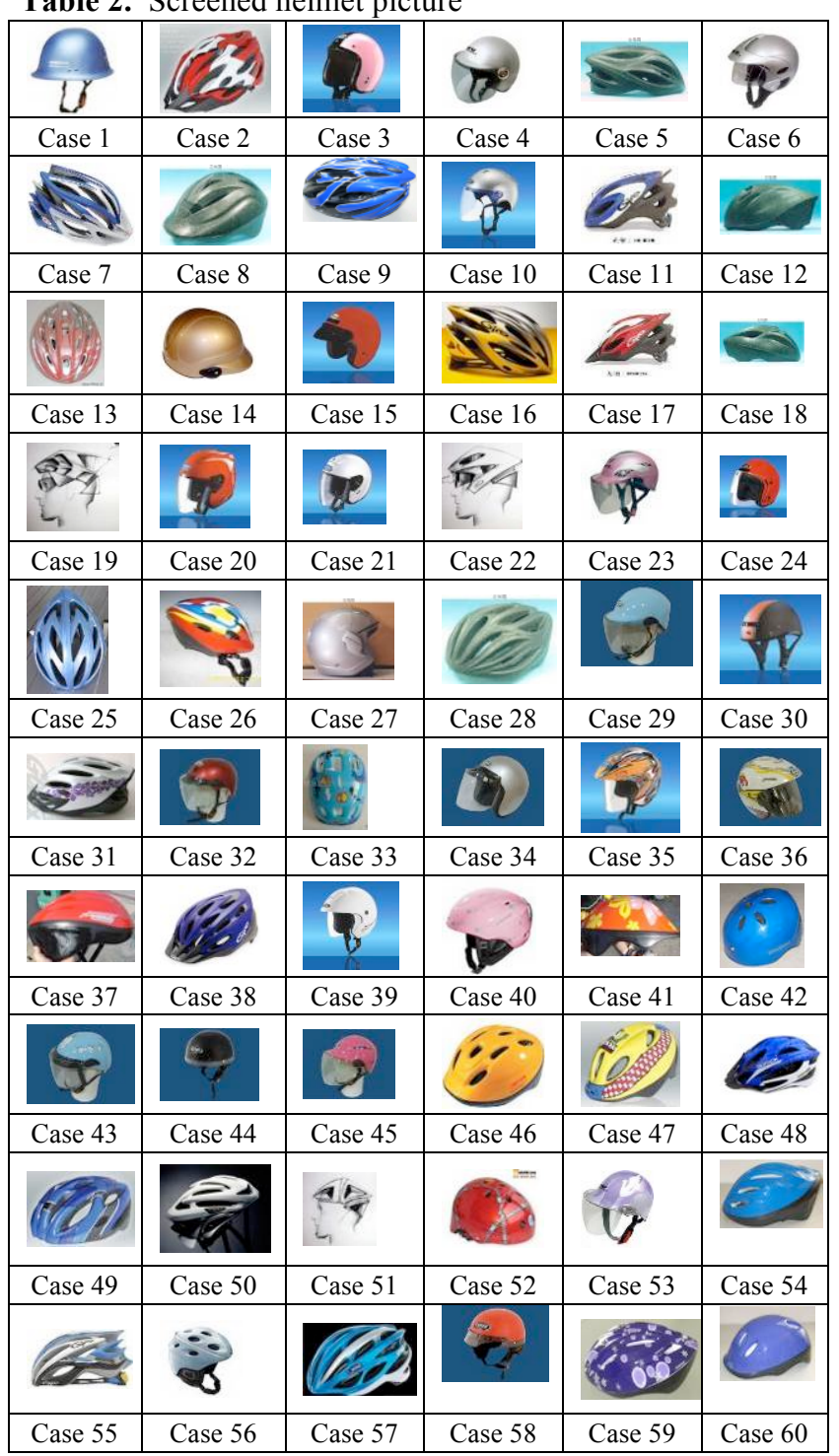

\section{Result analysis and discussion}

\subsection{Preliminary statistics of objects}

The survey was sent out, and 130 questionnaires were collected, including 117 valid questionnaires, with a 90\% effective rate. After the statistics of the 7-point rating scale was generated, the final survey model can be defined as follows.

$$
\left[\begin{array}{ccccc}
A_{11}^{K} & A_{12}^{K} & A_{13}^{K} & \ldots & A_{1 J}^{K} \\
A_{21}^{K} & A_{22}^{K} & A_{23}^{K} & \ldots & A_{2 J}^{K} \\
& \ldots & & A_{i j}^{K} & \\
A_{I 1}^{K} & A_{I 2}^{K} & A_{I 3}^{K} & \ldots & A_{I J}^{K}
\end{array}\right]
$$


Where $A_{i j}^{K}$ is the $\mathrm{j}$-th perceptual evaluation preference from $\mathrm{k}$ evaluators to the $\mathrm{i}$-th evaluation sample, $i$ is 60 , and $j$ is 12 .

The Leida criterion was first used to reject the abnormal perceptual image because the evaluation results reflected the sensation of most people. The mean value and variance of the $\mathrm{j}$-th perceptual evaluation preference to the $\mathrm{i}$-th evaluation sample are as follows.

$\overline{A_{i j}}=\sum_{K=1}^{K} A_{i j}^{K} / K$

$\sigma=\left[\sum_{K=1}^{K}\left(\overline{A_{i j}}-A_{i j}^{K}\right)^{2} /(K-1)\right]^{\frac{1}{2}}$

On the basis of the Leida criterion, we removed those satisfying $\left|\bar{A}_{i j}-A_{i j}^{K}\right|>3 \sigma$, and the evaluation value of most people is the remaining mean value. $\overline{A_{i j}}$ is the mean value of the $\mathrm{j}$-th perceptual description evaluation of the $\mathrm{i}$-th helmet form, which was written into a datasheet.

\subsection{Clustering analysis}

Clustering analysis is the method of studying the individual classification according to the characteristics of things. The principle is that individuals in the same category have great similarity and that individuals in different categories have great differences. Sample clustering, also known as Q-type clustering, is the clustering of observation. It is the classification of variable values that reflect the characteristics of observed objects. The samples were clustered using the SPSS software. In the statistics dialog box, the number of clustering was set to 12 categories. The dendrogram shows the clustering intuitively (see Fig. 1).With clustering analysis, the pictures were grouped into 12 types. After analyzing with helmet designers, we selected a representative helmet with a relatively simple form in each type. The form was simplified to establish a 3D model. Color interference was removed with renumbering (see Table 3)

Table 3. Survey sample diagram

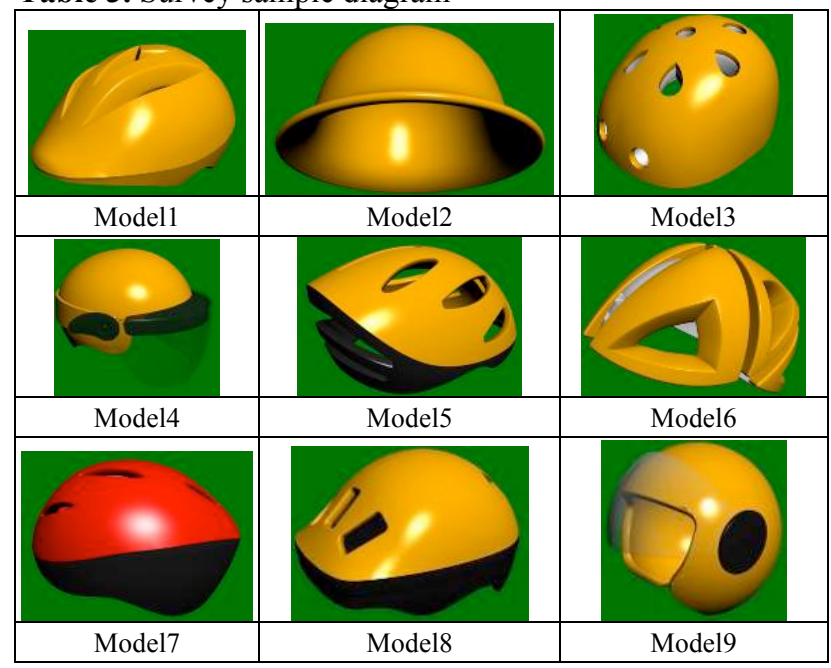

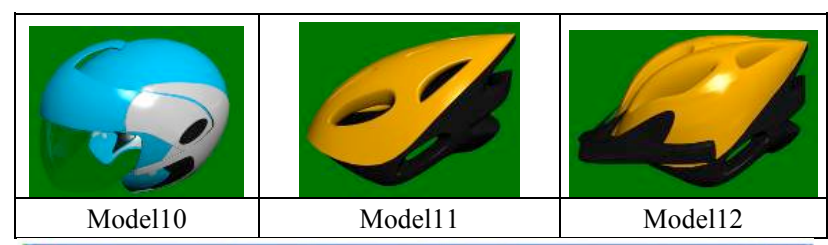
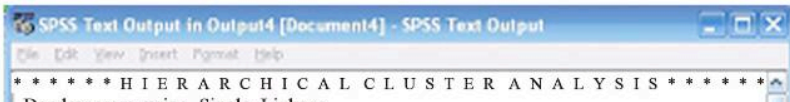
Dendrongram using Single Linkage

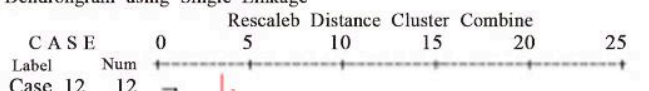

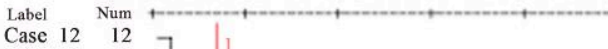

Case $18 \quad 18$

Case $8 \quad 8$

Case $41 \quad 41$

Case $60 \quad 60$

Case $46 \quad 46$

Case $26 \quad 26$

Case $37 \quad 37$

Case $49 \quad 49$

Case $25 \quad 25$

Case $31 \quad 31$

$\begin{array}{ll}\text { Case } 47 & 47 \\ \text { Case } 54 & 54\end{array}$

$\begin{array}{ll}\text { Case } 13 & 13\end{array}$

Case $59 \quad 59$

Case 59
Case 6

Case 56

Case $36 \quad 36$

Case $34 \quad 34$

Case $40 \quad 40$

Case $4 \quad 4$

$\begin{array}{ll}\text { Case } 27 & 27 \\ \text { Case } 35 & 35\end{array}$

Case $35 \quad 35$

Case $15 \quad 15$

Case $21 \quad 21$

Case $24 \quad 24$

Case 33

Case $20 \quad 20$

Case $23 \quad 23$

Case $14 \quad 14$

$53 \quad 53$

Case $32 \quad 32$

Case $45 \quad 45$

Case $10 \quad 10$

Case $17 \quad 17$

Case $38 \quad 38$

Case 2

Case $9 \quad 9$

Case $48 \quad 48$

Case $22 \quad 22$

$\begin{array}{ll}\text { Case } 51 & 51\end{array}$

Case $51 \quad 51$

Case $11 \quad 11$

Case $28 \quad 28$

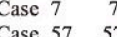

Case $16 \quad 16$

Case $55 \quad 55$

Case $50 \quad 50$

Case 5

Case 1

Case $44 \quad 44$

Case $58 \quad 58$

Case 30

Fig. 1. Tree diagram of clustering

\subsection{Multidimensional scaling analysis}

The study used multidimensional scaling (2D) of the scale in SPSS for analysis, with the scaling model of the Euclidean distance. The results generated scatter diagram of the samples in 2D space. The accumulation and dispersion of sample points are shown in Figs. 2 and 3.

The analysis results corroborated that, after four iterations, the improvement of the S-stress value was 0.00067 . The convergence criterion was achieved-less than the specified value of 0.001 . 


\begin{tabular}{|c|c|c|c|}
\hline \multirow[b]{2}{*}{$\begin{array}{l}\text { Stimulus } \\
\text { Number }\end{array}$} & \multirow[b]{2}{*}{$\begin{array}{l}\text { Stimulus } \\
\text { Nane }\end{array}$} & \multicolumn{2}{|c|}{ Dimension } \\
\hline & & 1 & 2 \\
\hline 1 & Model1 & .0578 & -.1417 \\
\hline 2 & Mode12 & .3917 & 1. 7199 \\
\hline 3 & Mode13 & -.0052 & -.0926 \\
\hline 4 & Model4 & 1. 5561 & .4012 \\
\hline 5 & Model5 & .0358 & -1.6464 \\
\hline 6 & Model6 & -2.0903 & .6855 \\
\hline 7 & Mode17 & .1358 & .0652 \\
\hline 8 & Mode18 & .3222 & .5951 \\
\hline 9 & Mode19 & 1.5109 & .3380 \\
\hline 10 & Mode110 & 1.0169 & -1.3000 \\
\hline 11 & Model11 & -1.3466 & -.8601 \\
\hline 12 & Model12 & -1.5851 & .2359 \\
\hline
\end{tabular}

Fig. 2. Scatter coordinates

4.4 Linear regression analysis

The mean values of perceptual words were regressed to the coordinates of the space map using multiple regressions. The direction of each perceptual vector represents the standardized regression coefficient of each regression line. Linear regression analysis was performed 12 times using the SPSS regression program to obtain standardized regression coefficients. Subsequently, the trigonometric function was used to calculate the angle between the attribute vector and the horizontal axis of the coordinate plane to generate the joint space map of the perceptual attribute vector and scattered points of helmets (see Fig. 3).

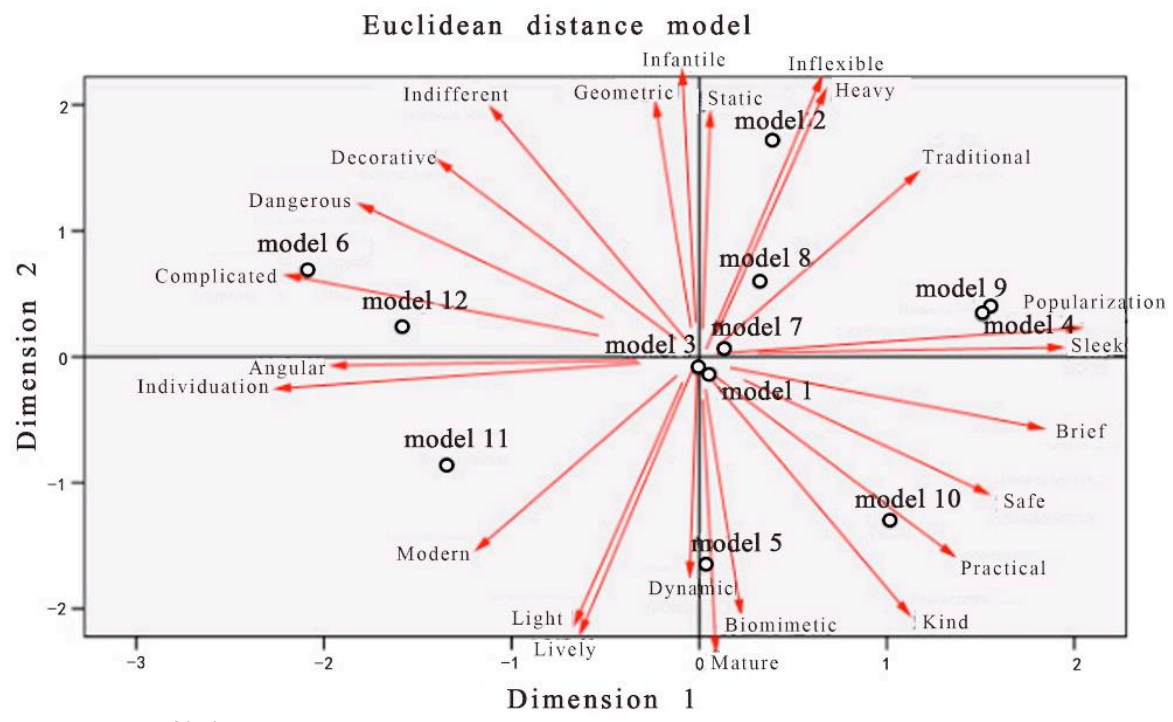

Fig. 3. Perceptual image space map of helmet

\subsection{Establishing semantic rule database of helmet form}

The correspondence between the helmet samples and perceptual words was calculated. According to the sample distance map (see Fig. 4) and the distance formula, we calculated the distance from the helmet sample point to the projected point of the perceptual vector and the origin to obtain the attribute value of the helmet sample. A high distance value indicated highly evident form features (See Table 4).

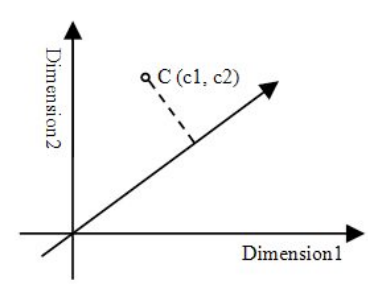

Fig. 4. Sample distance map

Table 4. Model attribute distance

\begin{tabular}{|c|c|c|c|c|c|c|c|c|c|c|c|c|}
\hline $\begin{array}{l}\text { Attribute } \\
\text { Sample }\end{array}$ & $\begin{array}{c}\text { Modern- } \\
\text { traditional }\end{array}$ & $\begin{array}{c}\text { Popularization- } \\
\text { individuation }\end{array}$ & $\mid \begin{array}{c}\text { Lively- } \\
\text { inflexible }\end{array}$ & $\begin{array}{l}\text { Practical- } \\
\text { decorative }\end{array}$ & $\begin{array}{l}\text { Practical- } \\
\text { decorative }\end{array}$ & $\begin{array}{l}\text { Mature- } \\
\text { infantile }\end{array}$ & $\begin{array}{l}\text { Light- } \\
\text { heavy }\end{array}$ & $\begin{array}{c}\text { Angular- } \\
\text { sleek }\end{array}$ & $\left|\begin{array}{c|}\text { Brief- } \\
\text { complicated }\end{array}\right|$ & $\begin{array}{c}\text { Dynamic- } \\
\text { static }\end{array}$ & $\begin{array}{c}\text { Safe- } \\
\text { dangerous }\end{array}$ & $\begin{array}{l}\text { Geometric- } \\
\text { biomimetic }\end{array}$ \\
\hline Model1 & -0.048 & 0.048 & -0.108 & 0.131 & 0.146 & 0.145 & -0.106 & 0.056 & 0.085 & -0.140 & 0.112 & 0.149 \\
\hline Model2 & 1.418 & 0.505 & 1.737 & -0.715 & -1.065 & -1.694 & 1.730 & 0.411 & 0.045 & 1.730 & -0.367 & -1.632 \\
\hline Model3 & -0.099 & -0.056 & -0.105 & 0.016 & 0.039 & 0.090 & -0.105 & -0.051 & -0.031 & -0.094 & -0.006 & 0.084 \\
\hline Model4 & 1.442 & 1.579 & 0.975 & 1.007 & 0.693 & -0.309 & 1.000 & 1.560 & 1.446 & 0.444 & 1.243 & -0.140 \\
\hline Model5 & -1.045 & -0.073 & -1.502 & 1.014 & 1.284 & 1.645 & -1.488 & 0.018 & 0.360 & -1.644 & 0.724 & 1.630 \\
\hline Model6 & -1.139 & -2.040 & -0.182 & -2.085 & -1.869 & -0.807 & \begin{tabular}{|l|}
-0.225 \\
\end{tabular} & -2.082 & -2.184 & 0.628 & -2.185 & -1.020 \\
\hline Model7 & 0.146 & 0.140 & 0.113 & 0.070 & 0.038 & -0.057 & 0.115 & 0.137 & 0.120 & 0.069 & 0.096 & -0.042 \\
\hline Model8 & 0.632 & 0.361 & 0.673 & -0.098 & -0.249 & -0.575 & 0.675 & 0.328 & 0.198 & 0.604 & 0.042 & -0.534 \\
\hline Model9 & 1.367 & 1.530 & 0.900 & 1.008 & 0.713 & -0.249 & 0.924 & 1.515 & 1.414 & 0.379 & 1.229 & -0.085 \\
\hline Model10 & -0.075 & 0.929 & -0.801 & 1.593 & 1.650 & 1.357 & \begin{tabular}{|l|}
-0.773 \\
\end{tabular} & 1.003 & 1.254 & -1.272 & 1.469 & 1.450 \\
\hline Model11 & -1.582 & -1.401 & -1.317 & -0.565 & -0.208 & 0.780 & \begin{tabular}{|l|}
-1.334 \\
\end{tabular} & -1.356 & -1.151 & -0.897 & -0.861 & 0.627 \\
\hline Model12 & -1.049 & -1.566 & -0.400 & -1.411 & -1.200 & -0.328 & -0.430 & -1.582 & -1.600 & 0.192 & -1.537 & -0.493 \\
\hline
\end{tabular}

We validated that only the absolute attribute values of some perceptual words in model 6 are greater than 2. For instance, the absolute value of the "personalized" attribute value was 2.040 , that of the "decorated" attribute value was 
2.085 , that of the "angular" attribute value was 2.082 , that of the "complex" attribute value was 2.184 , and that of the "dangerous" attribute value was 2.185. These scenarios verify that the form style of model 6 had the perceptual characteristics of these words. However, the absolute attribute value of the perceptual words in model 3 was less than 0.1 , indicating that the form characteristics were not apparent for model 3 .

According to the size of the absolute value of the distance, the correspondence table between the image words and helmets was obtained (see Table 5) and used as the original data in the semantic rule system database of helmet form.

\section{Conclusions}

To establish the mapping relationship between product form and perceptual image, this study applied Kansei engineering, semantic difference method, and multidimensional scaling method from users' cognitions. The algorithm model for perceptual quantification of the helmet form was proposed. The following conclusions could be drawn:

(1) The perceptual attribute value of the product sample is the distance from the sample point to the projection point of perceptual vector and the origin, which determines the correlation between the sample and the perceptual vector.
(2) An absolute perceptual attribute value of the sample indicates that the sample form has considerable perceptual characteristics of the vector.

(3) A short distance between the samples indicates the significant relevance of the image design of the sample forms.

The algorithm in this study can establish the preference prediction model of users to help designers accurately grasp users' perceptual needs of product forms, thereby making correct and efficient decision-making in product development and design positioning. In the perceptual design database, we can find samples and related attribute values of each perceptual feature. However, it does not have reference value for design until the absolute sample attribute value is greater than the critical value. Therefore, the determination of the perceptual semantic threshold should be explored further.

\section{Acknowledgments}

This study was supported by the science and technology planning project of Baoding (Grant No. 12ZG027).

This is an Open Access article distributed under the terms of the Creative Commons Attribution License.

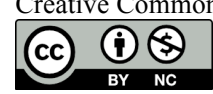

\section{References}

1. Su J. N., Wang p., Zhang S.T., "Review of Key Technologies of Product Image Styling Design". Journal of Machine Design, 30(1), 2013, pp. 97-100.

2. Luo S. J., Pan Y. H., "Review of Theory, Key Technologies and Its Application of Perceptual Image in Product Design". Journal of Mechanical Engineering, 43(3), 2007, pp. 8-13.

3. ISHIHARA S, ISHIHARA K, NAGAMACHI M., "Analysis of Kansei Structure on Shoes Using Self-Organizing Neural Network". International Journal of Industrial Ergo-nomics, 19(96), 1997, pp.93-104.

4. Markus Hartono., "The Extended Integrated Model of Kansei Engineering, Kano, and TRIZ Incorporating Cultural Differences into Services". International Journal of Technology, 1, 2016, pp.97-104.

5. Pierre Lévy., "Beyond Kansei Engineering: The Emancipation of Kansei Design". International Journal of Design, 7(2) ,2013, pp.83.

6. AKINORI H, TAKAMASA S., "A Kansei Engineering Approach to a Driver/vehicle System". International Journal of Industrial Ergonomics, 15(1), 1995 ,pp.25-37.

7. Yang Y. S., "Application of Positive Kansei Engineering in the Office Products Design”. Packaging Engineering, 38(16), 2017, pp.113-118.

8. A.M. Lokman, M.B.C. Haron, S.Z.Z. Abidin, N.E.Z. Khalid, S. Ishihara., "Prelude to Natphoric Kansei Engineering Framework". Journal of Software Engineering and Applications, 6, 2013, pp.638-644.

9. Zhao Y. Y., Bian F., Li J. T., "Image Research of Product Material Based on Kansei Engineering". Journal of Machine Design, 8, 2015, pp. 117-121.

10. Xi L., Zhang H., Chen J. H., "Modeling design of tourist service robot based on Fuzzy-Kano model". Journal of Machine Design, 32(8), 2017, pp.110-113.
11. Ana Hadiana., "Design of Kansei Engineering Support System Using Fuzzy Multiple Attribute Decision Making”. International Journal of Basic and Applied Science, 5(1), 2016, pp.24-30.

12. Wang K., Han J. X., "Applying Kansei Engineering to Decision Making in Chinese Wine bottle Form Design". Packaging Engineering, 38(14), 2017, pp.229-234.

13. Du Y., Zhang Y.R., Haung X.L.,"Studyof Backpack form Basedonkansei Engineering". Industrial Design, 3, 2017, pp.6162.

14. Guo B. j., Xue Y., "Emotionl Evaluation OF Cheongsam's, Collar Shapes and Open Front". Journal of xian polytechniic university, 31(5)2017, pp.338-344.

15. Kuang J., Jiang P., "Product platform design for a product family based on Kansei engineering". Journal of Engineering design, 20 (6), 2009, pp.589-607.

16. J. Kim, J. Lee, D. Choi., "Designing emotionally evocative homepages: an empirical study of the quantitative relations between design factors and emotional dimensions". International Journal of Human-Computer Studies, 59 (6), 2003, pp. 899-940.

17. C. Barnes, S. P. Lillford, "Decision support for the design of affective products". Journal of Engineering Design, 20(5), 2009, pp.477-92.

18. Du H. M., "Evaluation of Product Design Based on Kansei Engineering and FAHP". Journal of Xi'an Technological University, 3, 2014, pp.244-249.

19. H. You, T. Ryu, K. Oh, M. H. Yun, K. J. Kim., "Development of Customer Satisfaction Models for Automotive Interior Materials". International Journal of Industrial Ergonomics, 36 (4), 2006, pp.323-30. 